\title{
Analytical investigation of poorly damped conditions in VSC-HVDC systems
}

\author{
Yujiao Song, Claes Breitholtz, Georgios Stamatiou, and Massimo Bongiorno
}

\begin{abstract}
In this paper, strong AC-grid connected VSCHVDC systems are studied. Under specific conditions, such systems can suffer from both stability and poor damping related issues, which warrants a stability study. Analytical eigenvalue expressions can directly demonstrate the impact of physical or control parameters on the system stability. However, especially in case of high-order systems, such expressions are challenging to obtain. This paper suggests a method to symbolically represent approximative eigenvalues of two-terminal VSC-HVDC systems, which could also be used to analyze the system dynamics. In addition, by applying symbolic-isolation method, the order of a multi-terminal VSC-HVDC system can be reduced to an equivalent two-terminal VSC-HVDC system, which enables the proposed method to provide symbolic pole expressions. Numerical studies based on Matlab simulations are presented, showing the accuracy of the analytical eigenvalue expressions and providing useful hints on the impact of physical or control parameters.
\end{abstract}

\section{INTRODUCTION}

Voltage source converter based high voltage direct current transmission (VSC-HVDC) systems have now been in operation since 1997 as they have offered a breakthrough in the controllability and stabilization of electric power transmission systems [1]. However, dynamic interaction between the system components increases the risk of potential poorlydamped conditions or even instability. Such occurrences have been described as e.g. oscillations caused by HVDC terminals [2], [3] or instabilities in DC power systems [4], [5].

Poorly-damped resonances between converter stations and DC cables can appear both in two-terminal and multiterminal VSC-HVDC. Such problems are typically approached by using numerical analysis to determine the actual values of the systems poles [6]. However, acquiring analytical expressions for these poles has the benefit of better understanding in which selected parameters of the system can affect the frequency and damping characteristics of its eigenvalues. Hence, it is valuable if such symbolic descriptions can be obtained for a poorly-damped VSCHVDC link, highlighting the relationship between the system parameters and its poorly-damped poles.

This work was supported by Chalmers Energy Initiative

Y. Song and C. Breitholtz are affiliated to the department of Signals and Systems, division of Automatic Control, Automation and Mechatronics, group of Automatic Control, Chalmers University of Technology, SE-412 96, Gothenburg, Sweden yujiao@chalmers.se; claesbrechalmers.se

G. Stamatiou and M. Bongiorno are with the department of Energy and Environment, division of Electric Power Engineering, Chalmers University of Technology, SE-412 96, Gothenburg, Sweden georgios.stamatiou@chalmers.se; massimo.bongiorno@chalmers.se
Relevant research is to be found mostly in traction systems as in [7], where a rectifier and an inverter are connected via DC lines. However, the analytical description of the system poles only considers the resonance of the DC cable, disregarding the effect of the converter controllers. The analytical eigenvalues of the DC-link in a two-terminal VSCHVDC connection is provided in [8], but is only applicable for zero power transfer. Approximate symbolic eigenvalues in multi-terminal VSC-HVDC (MTDC) are also provided in [9] but significant simplifications are required, influencing the validity of the final solutions.

The aim of this paper is to propose a method to efficiently characterize poorly-damped conditions that may appear on the DC-side of a two-terminal VSC-HVDC link, while the interaction with the $\mathrm{AC}$-side is neglected considering the presence of strong AC grids. The focus is to analytically describe the poles of the system, in terms of characteristic frequency and damping. Consequently, the interaction between the controller parameters of the terminals and the physical properties of the DC-link can be better understood and evaluated.

For MTDC system, it is assumed that one converter is controlling the DC-voltage and the rest of the converters are controlling the transmitted active power between the AC- and DC-sides. The DC-voltage controlled VSC keeps symbolic physical and controller parameters, while the remaining converters and DC-grid parameters are numerically known. The symbolic-isolation method [10] is applied to reduce the system order into four and thus enables the proposed method to derive the approximate eigenvalues of the system in symbolic expressions.

\section{TWO-TERMINAL VSC-HVDC SYSTEM}

The two-terminal VSC-HVDC system is described by Fig.1. It consists of two VSC stations, where each of them is assumed to be connected to a strong AC grid. At the AC-side of the VSCs, the series inductances $\left(L_{1}, L_{2}\right)$ and resistances $\left(R_{1}, R_{2}\right)$ represent the $\mathrm{AC}$ reactor and the power losses in the converter. The shunt capacitors at the DC-side are denoted by $C_{c 1}, C_{c 2}$ respectively. In this paper, the DC cable would be interpreted as a single $\Pi$-section, which is proved to be sufficient for system stability evaluation [11].

In standard VSC-HVDC systems, one converter (station 1) is assigned the duty of DC-voltage controller to secure the stability of the DC-bus voltage; the other converter (station 2) serves as the active power controller to guarantee and balance the power exchanges [12]. 


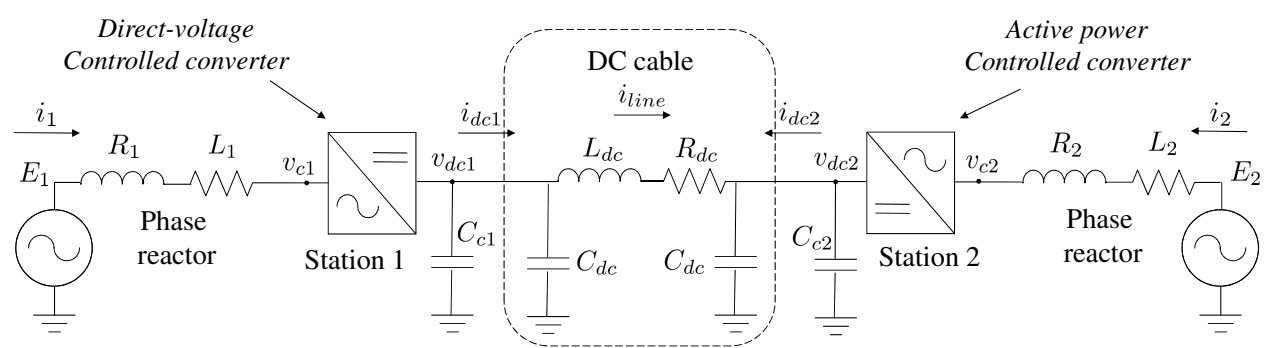

Fig. 1: Two-terminal point-to-point VSC-HVDC system embedded in a strong AC-environment. $E$ is the point of common coupling (PCC) voltage; $i$ is the converter input current; $v_{c}$ is the converter voltage; $v_{d c}$ is the DC voltage at the terminal; $i_{d c}$ is the DC current which flows into the DC grid from converter; $i_{\text {line }}$ is the DC current passing through the DC cable inductance and resistance.

The local controller of the VSC is separated into two cascaded parts: inner current controller and outer controller. The inner current controller provides the converter voltage reference $\left(v_{c d}^{r e f}\right)$ and $\left(v_{c q}^{r e f}\right)$ to the pulse width modulator (PWM). The $d$-axis outer controller is used to track the reference of either DC voltage or active power and generates the $d$-axis current reference $\left(i_{d}^{\text {ref }}\right)$ to the inner current loop. The $q$-axis outer controller is used to limit the voltage drop of the PCC voltage, generating the $q$-axis current reference $\left(i_{q}^{\text {ref }}\right)$ to the inner current loop as well [13].

\section{A. Statements and Assumptions}

The main statements and assumptions of the proposed model are the following:

- The reference signal of $x$ is $x^{r e f}$ and the steady state of $x$ is $x_{0}$.

- The differential operator is expressed by $p$ and the Laplace transform of a time domain variable $x(t)$ is denoted by $\tilde{x}(s)$.

- The connected AC grids are well balanced and strong, i.e. the nominal $\mathrm{AC}$ grid voltage is as most subject to small variation (i.e. $\Delta E_{d} \approx 0, \Delta E_{q} \approx 0$ ) and the dynamics of Phase Locked Loop (PLL) and low pass filter for feed forward PCC voltage are ignored.

- The model is derived in the so-called $d q$ reference frame, which is chosen to be aligned with the AC voltage direction by robust PLL, i.e. $E_{q k 0}=0, k=1,2$. $E_{q k 0}$ is the steady state of the $q$-axis PCC voltage [14].

- Since the $q$-axis current $\left(i_{q}\right)$ has no impact on the dynamics at the DC-side (after $d$ - and $q$-dynamics decoupling), $i_{q}^{r e f}$ is thus assumed to be zero.

- The inner current loop is designed to be at least ten times faster than the outer loop, which enables the inner current loop to be interpreted as infinitely fast.

- The active power controlled VSC is assumed to be operated under steady state. Otherwise, the dynamics caused by the AC-side of the active power controlled VSC is considered as an disturbance input to the DCside dynamics.

- The VSCs are assumed to be symmetric, in this section, the variables and parameters would not be subscribed by the number 1 and 2 which are used to denote different VSCs.

\section{B. DC-voltage controlled VSC}

As claimed above, the local controller of a VSC is separated into two cascaded parts: inner current controller and outer controller. The inner current controller is a PIcontroller with PCC voltage feed-forward and cancelation of the $d q$ cross coupling [15]. The PI-controller parameters are designed by pole cancelation, which speeds up the inner current dynamics to be ten times faster than the outer loop. Consequently, the linearized inner current closed loop in the Laplace domain is [16]:

$$
\begin{aligned}
\Delta \tilde{i}_{d}(s) & =\frac{a_{c}}{s+a_{c}} \Delta \tilde{i}_{d}^{r e f}(s)=g_{c}(s) \Delta \tilde{i}_{d}^{r e f}(s) \\
\Delta \tilde{i}_{q}(s) & =\frac{a_{c}}{s+a_{c}} \Delta \tilde{i}_{q}^{r e f}(s)
\end{aligned}
$$

Since the connected AC grid is assumed to be strong, there is no need to limit the voltage drop of the PCC voltage, i.e. $\Delta i_{q}^{r e f}=0$.

The DC voltage outer controller is a PI-controller with DC load power feed-forward. The linearized DC voltage dynamics and the controllers are [16]:

$$
\begin{aligned}
\frac{\mathrm{d} \Delta v_{d c}}{\mathrm{~d} t} & =\left(\Delta P-\Delta P_{\text {load }}\right) / C_{c} / v_{d c 0} \\
\Delta P & =E_{0} \Delta i_{d}+i_{d 0} \Delta E_{d}+i_{q 0} \Delta E_{q} \approx E_{0} \Delta i_{d} \\
\Delta P_{\text {load }} & =v_{d c 0} \Delta i_{d c}+i_{d c 0} \Delta v_{d c} \\
\Delta P^{r e f} & =K_{p}\left(1+\frac{1}{T_{i} \cdot p}\right)\left(\Delta v_{d c}^{r e f}-\Delta v_{d c}\right)+F(p) \Delta P_{\text {load }} \\
& =E_{0} \Delta i_{d}^{r e f} \\
F(s) & =\frac{a_{f}}{s+a_{f}}
\end{aligned}
$$

After choosing $K_{p}=a_{d} v_{d c 10} C_{c}$, the closed-loop DC voltage dynamics is:

$\Delta \tilde{v}_{d c}=\frac{a_{d} g_{c}\left(1+\frac{1}{T_{i} s}\right)}{s+a_{d} g_{c}\left(1+\frac{1}{T_{i} s}\right)} \Delta \tilde{v}_{d c}^{r e f}+\frac{F(s) g_{c}(s)-1}{C_{c} v_{d c 10}\left[s+a_{d} g_{c}\left(1+\frac{1}{T_{i} s}\right)\right]} \Delta \tilde{P}_{\text {load }}$

From a control point of view, the DC-load power represents a disturbance which can be compensated by the power feed-forward. Therefore, the zero steady-state error can be reached by a P-controller. However, if losses on the valves of the VSC are considered, integral-action should be added. In the present analysis, the losses are neglected and 
the simplified DC-voltage dynamics after assuming $T_{i} \rightarrow \infty$, $a_{c} \rightarrow \infty$ is:

$$
\Delta \tilde{v}_{d c}=\frac{a_{d}}{s+a_{d}} \Delta \tilde{v}_{d c}^{r e f}+\frac{F(s)-1}{C_{c} v_{d c 10}\left(s+a_{d}\right)} \Delta \tilde{P}_{l o a d}
$$

\section{Active power controlled VSC}

For the outer controller of transmitted active power, again a PI-controller is used. The controller parameters are designed by inner current loop pole cancelation. The linearized active power and DC voltage expressions are [16]:

$$
\begin{aligned}
\Delta \tilde{P} & =\frac{a_{p}}{s+a_{p}} \Delta \tilde{P}^{r e f} \\
\frac{\mathrm{d} \Delta v_{d c}}{\mathrm{~d} t} & =\left(\Delta P-\Delta P_{\text {load }}\right) / C_{c} / v_{d c 0}
\end{aligned}
$$

Under steady state, i.e. $\Delta P=0$, the active power controlled VSC is equivalent to a shunt RC-circuit where the resistance is $v_{d c 0} / i_{d c 0}$ and the capacitance is $C_{c}$. It is worth to be mentioned that the resistance would be negative as the active power is transmitted from the DC- to the AC-side. The simplified DC-voltage dynamics is:

$$
\frac{\mathrm{d} \Delta v_{d c}}{\mathrm{~d} t}=-\frac{i_{d c 0}}{C_{c} v_{d c 0}} \Delta v_{d c}-\frac{1}{C_{c}} \Delta i_{d c}
$$

\section{DC cable}

Since the single $\Pi$-section DC cable model is proved to be sufficient for VSC-HVDC system stability evaluation [11], the DC cable dynamics can be expressed as:

$$
\begin{aligned}
\frac{\mathrm{d} \Delta i_{\text {line }}}{\mathrm{d} t} & =\frac{1}{L_{d c}} \Delta v_{d c 1}-\frac{1}{L_{d c}} \Delta v_{d c 2}-\frac{R_{d c}}{L_{d c}} \Delta i_{\text {line }} \\
\frac{\mathrm{d} \Delta v_{d c 1}}{\mathrm{~d} t} & =\frac{1}{C_{d c}} \Delta i_{d c 1}-\frac{1}{C_{d c}} \Delta i_{\text {line }} \\
\frac{\mathrm{d} \Delta v_{d c 2}}{\mathrm{~d} t} & =\frac{1}{C_{d c}} \Delta i_{d c 2}+\frac{1}{C_{d c}} \Delta i_{\text {line }}
\end{aligned}
$$

\section{E. State space representation}

Combining the dynamics of DC-voltage controlled VSC (8), active power controlled VSC (11) and DC cable (12)(14), the two-terminal VSC-HVDC system can be interpreted as a fourth order state space model, where $C_{t o t}=C_{c}+C_{d c}$ (due to that the converters are assumed to be symmetric) and $\Delta i_{\text {load }}^{f}$ is the filtered load power normalized by $v_{d c 10}$, i.e. $\Delta i_{\text {load }}^{f}=F(p) \Delta P_{\text {load }} / v_{d c 10}$ :

$$
\begin{aligned}
& \frac{\mathrm{d}}{\mathrm{d} t} x=A_{s} \cdot x+B_{s} \cdot \Delta v_{d c 1}^{r e f} \\
& y=C_{s} \cdot x=\left[\begin{array}{llll}
0 & 1 & 0 & 0
\end{array}\right] \cdot x=\Delta v_{d c 1} \\
& x^{T}=\left[\begin{array}{llll}
\Delta i_{\text {load }}^{f} & \Delta v_{d c 1} & \Delta i_{\text {line }} & \Delta v_{d c 2}
\end{array}\right] \\
& B_{s}^{T}=\left[\begin{array}{llll}
a_{f} a_{d} \frac{C_{d c} C_{c}}{C_{t o t}} & a_{d} \frac{C_{c}}{C_{t o t}} & 0 & 0
\end{array}\right]
\end{aligned}
$$$$
A_{s}=\left[\begin{array}{cccc}
-a_{f} \frac{C_{c}}{C_{t o t}} & a_{f} \frac{C_{c} i_{d c 10}-a_{d} C_{d c} C_{c} v_{d c 10}}{C_{t o t} v_{d c 10}} & a_{f} \frac{C_{c}}{C_{t o t}} & 0 \\
\frac{1}{C_{t o t}} & -\frac{a_{d} C_{c} v_{d c 1}+i_{d c 10}}{C_{t o t} v_{d c 10}} & -\frac{1}{C_{t o t}} & 0 \\
0 & \frac{1}{L_{d c}} & -\frac{R_{d c}}{L_{d c}} & -\frac{1}{L_{d c}} \\
0 & 0 & \frac{1}{C_{t o t}} & -\frac{P_{20}}{C_{t o t} v_{d c 20}^{2}}
\end{array}\right]
$$

It is easy to prove that the state space model is minimum order, implying that the system poles are the eigenvalues of the state matrix $A_{s}$. The suggested method to analytically describe the poles of the system will thus operate on the symbolic state matrix $A_{s}$, in an effort to extract the desired analytical approximate expressions.

\section{SYMBOLIC EIGENVALUES}

The characteristic polynomial of the $4 \times 4$ matrix $A_{s}$ can be seen as a product of two second order polynomials $p_{1}(\lambda)$ and $p_{2}(\lambda)$. We are in fact most interested in $p_{1}(\lambda)$, which is poorly damped and its roots are strongly dependent of the DC-cable length. $p_{2}(\lambda)$, whose roots are well damped and may even be real, is to a low extent dependent of the DC-cable length.

Letting the DC-cable length approaches zero, the fourth order system will exactly reduce to a second order system. The characteristic polynomial of the new $2 \times 2$ state matrix $A_{s 2}$ can be assumed to approximate the well damped polynomial $p_{2}(\lambda)$, i.e. $p_{2}(\lambda) \approx \tilde{p}_{2}(\lambda)$. Therefore, we can use $\tilde{p}_{2}(\lambda)$ to calculate the poorly damped polynomial $p_{1}(\lambda)$.

To reduce the visual complexity, $A_{s}$ is re-written as:

$$
A_{s}=\left[\begin{array}{cccc}
-a & b & a & 0 \\
\frac{1}{C_{t o t}} & -d & -\frac{1}{C_{t o t}} & 0 \\
0 & \frac{1}{L_{d c}} & -\frac{R_{d c}}{L_{d c}} & -\frac{1}{L_{d c}} \\
0 & 0 & \frac{1}{C_{t o t}} & e
\end{array}\right]
$$

$$
\begin{aligned}
\text { where } a & =a_{f} \frac{C_{c}}{C_{t o t}}, b=a_{f} \frac{C_{c} i_{d c 10}-a_{d} C_{d c} C_{c} v_{d c 10}}{C_{t o t} v_{d c 10}}, \\
d & =\frac{a_{d} C_{c} v_{d c 10}+i_{d c 10}}{C_{t o t} v_{d c 10}}, e=-\frac{P_{20}}{C_{t o t} v_{d c 20}^{2}}
\end{aligned}
$$

A. Well damped polynomial $p_{2}(\lambda)$

If the DC-cable length is zero, then $x_{2}=x_{4}, \dot{x}_{2}=\dot{x}_{4}$ and $\dot{x}_{3}$ is removed. The new second order autonomous state space model is:

$$
\left[\begin{array}{l}
\dot{x}_{1} \\
\dot{x}_{2}
\end{array}\right]=\left[\begin{array}{cc}
-\frac{a}{2} & b-\frac{a C_{t o t}}{2}(d+e) \\
\frac{1}{2 C_{t o t}} & -\frac{d-e}{2}
\end{array}\right]\left[\begin{array}{l}
x_{1} \\
x_{2}
\end{array}\right]=A_{s 2}\left[\begin{array}{l}
x_{1} \\
x_{2}
\end{array}\right]
$$

The characteristic function of $A_{s 2}$ is:

$$
\begin{aligned}
\tilde{p}_{2}(\lambda) & =\operatorname{det}\left(\lambda I-A_{s 2}\right) \\
& =\lambda^{2}+0.5(a+d-e) \lambda+0.5\left(a d-b / C_{t o t}\right)
\end{aligned}
$$

\section{B. Poorly damped polynomial $p_{1}(\lambda)$}

The characteristic polynomial of the fourth order state matrix $A_{s}$ is given below, where $k_{1}$ and $k_{2}$ are the first and second order coefficient respectively:

$$
\begin{aligned}
\operatorname{det}\left(\lambda I-A_{s}\right)= & \lambda^{4}+\left(a+d-e+\frac{R_{d c}}{L_{d c}}\right) \lambda^{3}+k_{2} \lambda^{2}+k_{1} \lambda+ \\
& +\left(a d-\frac{b}{C_{t o t}}\right)\left(\frac{1}{L_{d c} C_{t o t}}-e \frac{R_{d c}}{L_{d c}}\right)
\end{aligned}
$$

Let $\lambda_{1,2}$ be the roots of polynomial $p_{1}(\lambda)$ and $\lambda_{3,4}$ be the roots of polynomial $p_{2}(\lambda)$. They satisfy:

$$
\begin{aligned}
\lambda_{1}+\lambda_{2}+\lambda_{3}+\lambda_{4} & =-\left(a+d-e+\frac{R_{d c}}{L_{d c}}\right) \\
\lambda_{1} \cdot \lambda_{2} \cdot \lambda_{3} \cdot \lambda_{4} & =\left(a d-\frac{b}{C_{t o t}}\right)\left(\frac{1}{L_{d c} C_{t o t}}-e \frac{R_{d c}}{L_{d c}}\right)
\end{aligned}
$$


TABLE I: Parameters of VSC-HVDC system

\begin{tabular}{|l|c|c|}
\hline Cable distance & length & $100 \mathrm{~km}$ \\
\hline Cable inductance density & 1 & $0.316 \mathrm{mH} / \mathrm{km}$ \\
\hline Cable capacitance density & $\mathrm{c}$ & $0.138 \mu \mathrm{F} / \mathrm{km}$ \\
\hline Cable resistance density & $\mathrm{r}$ & $0.03 \Omega / \mathrm{km}$ \\
\hline DC shunt capacitor $\left(\tau_{s}=4.1 \mathrm{~ms}\right)$ & $C_{1}=C_{2}$ & $20 \mu \mathrm{F}$ \\
\hline Rated DC voltage & $v_{d c 0}$ & $640 \mathrm{kV}$ \\
\hline Rated transmission power & $P_{b}$ & $1000 \mathrm{MW}$ \\
\hline Bandwidth of DC-voltage controller & $a_{d}$ & $300 \mathrm{rad} / \mathrm{s}(0.95$ p.u. $)$ \\
\hline Bandwidth of DC-load power filter & $a_{f}$ & $300 \mathrm{rad} / \mathrm{s}(0.95$ p.u. $)$ \\
\hline
\end{tabular}

Combining (21)-(22) with the approximated polynomial $\tilde{p}_{2}(\lambda)(19)$, it is known that:

$$
\begin{aligned}
\lambda_{3}+\lambda_{4} & \approx-\frac{1}{2}(a+d-e) ; \quad \lambda_{3} \cdot \lambda_{4} \approx \frac{1}{2}\left(a d-\frac{b}{C_{t o t}}\right) \\
\Rightarrow \lambda_{1}+\lambda_{2} & =-\frac{1}{2}\left(a+d-e+2 \frac{R_{d c}}{L_{d c}}\right) \\
\lambda_{1} \cdot \lambda_{2} & =2\left(\frac{1}{L_{d c} C_{t o t}}-e \frac{R_{d c}}{L_{d c}}\right)
\end{aligned}
$$

The approximate poorly damped polynomial is:

$$
\begin{aligned}
p_{1}(\lambda) & \approx\left(\lambda-\lambda_{1}\right)\left(\lambda-\lambda_{2}\right) \\
& \approx \lambda^{2}+\frac{1}{2}\left(a+d-e+2 \frac{R_{d c}}{L_{d c}}\right) \lambda+2\left(\frac{1}{L_{d c} C_{t o t}}-e \frac{R_{d c}}{L_{d c}}\right)
\end{aligned}
$$

\section{Accuracy of the approximations}

In this section, the eigenvalues $\lambda_{\text {num }}$ of the VSC-HVDC system, found be numerical extracting them from $A_{s}$, are compared to the symbolic eigenvalues $\lambda_{\text {sym }}$ expressed by (25), (19). Different scenarios are investigated where the values of all the system's parameters and steady-state entries are set to be constantly equal to the values of Tab. I, with the exception of one parameter that is allowed to vary. The motive for doing so is to observe the accuracy of the analytical expressions compared to the numerical eigenvalues, for different values of the selected parameters. Five scenarios are considered.

1 Variation of $a_{f}$ between 10-600 rad/s.

2 Variation of $a_{d}$ between 10-600 rad/s.

3 Variation of $a_{d}=a_{f}$ between $10-600 \mathrm{rad} / \mathrm{s}$.

4 Variation of the cable length between $10-600 \mathrm{~km}$.

5 Variation of the active power transmitted at station 2 between -1000-0 MW.

The nominal algebraic error is shown in Tab. II. The first four columns show the nominal algebraic error of the real and imaginary part respectively:

$$
\begin{aligned}
\varepsilon_{\text {real }} & =\left|\left(\operatorname{Re}\left[\lambda_{\text {num }}\right]-\operatorname{Re}\left[\lambda_{\text {sym }}\right]\right) / \operatorname{Re}\left[\lambda_{\text {num }}\right]\right| \cdot 100 \% \\
\varepsilon_{\text {imag }} & =\left|\left(\operatorname{Im}\left[\lambda_{\text {num }}\right]-\operatorname{Im}\left[\lambda_{\text {sym }}\right]\right) / \operatorname{Im}\left[\lambda_{\text {num }}\right]\right| \cdot 100 \%
\end{aligned}
$$

The last two columns show the nominal algebraic error of the pole magnitude, which is the same as the difference of the system natural frequency for complex poles:

$$
\begin{aligned}
\varepsilon_{\text {mag }} & =|| \lambda_{\text {num }}|-| \lambda_{\text {sym }}|/| \lambda_{\text {num }}|| \cdot 100 \% \\
& =\left|\left(\omega_{n, \text { num }}-\omega_{n, \text { sym }}\right) / \omega_{n, \text { num }}\right| \cdot 100 \%
\end{aligned}
$$

The comparison result shows that the maximum error appears in the real part of scn. 4, which can be decreased
TABLE II: Numerical difference between eigenvalues of $A_{s}$ and the roots of $p_{1}(\lambda), p_{2}(\lambda)[\%]$

\begin{tabular}{|c|c|c|c|c|c|c|}
\hline \multirow{2}{*}{ Scenarios } & \multicolumn{2}{|c|}{ Real } & \multicolumn{2}{c|}{ Imaginary } & \multicolumn{2}{c|}{ Magnitude } \\
\cline { 2 - 7 } & Max & Average & Max & Average & Max & Average \\
\hline Scn. 1 & 0.86 & 0.59 & 1.55 & 0.76 & 1.51 & 0.72 \\
\hline Scn. 2 & 0.86 & 0.59 & 1.55 & 0.74 & 1.51 & 0.72 \\
\hline Scn. 3 & 2.64 & 1 & 2.43 & 0.84 & 2.26 & 0.8 \\
\hline Scn. 4 & 8.05 & 4.02 & 4.04 & 1.25 & 4.27 & 1.34 \\
\hline Scn. 5 & 0.75 & 0.74 & 0.84 & 0.81 & 0.82 & 0.8 \\
\hline
\end{tabular}

a) The pole pair $\lambda_{1,2}$ that has larger ratio of $\operatorname{Im}\{\lambda\} / \operatorname{Re}\{\lambda\}$

\begin{tabular}{|c|c|c|c|c|c|c|}
\hline \multirow{2}{*}{ Scenarios } & \multicolumn{2}{|c|}{ Real } & \multicolumn{2}{c|}{ Imaginary } & \multicolumn{2}{c|}{ Magnitude } \\
\cline { 2 - 7 } & Max & Average & Max & Average & Max & Average \\
\hline Scn. 1 & 1.16 & 0.85 & 3.15 & 1.8 & 1.48 & 0.73 \\
\hline Scn. 2 & 1.16 & 0.85 & 3.15 & 1.8 & 1.48 & 0.73 \\
\hline Scn. 3 & 5.33 & 1.58 & 5.12 & 2.03 & 2.21 & 0.79 \\
\hline Scn. 4 & 20.63 & 8.49 & 2.06 & 1.21 & 4.46 & 1.37 \\
\hline Scn. 5 & 1.09 & 1.07 & 1.53 & 0.96 & 0.61 & 0.24 \\
\hline
\end{tabular}

b) The pole pair $\lambda_{3,4}$ that has smaller ratio of $\operatorname{Im}\{\lambda\} / \operatorname{Re}\{\lambda\}$

from $20.63 \%$ to $7.42 \%$ if the studied maximum cable length decreases from $600 \mathrm{~km}$ to $300 \mathrm{~km}$. Except of scn. 4, the errors of the approximated result are bounded by $5.33 \%$ and in average are lower than $2.03 \%$. In general, the method provide compact expressions that closely fit the numerically calculated eigenvalues of the system, with relatively small error.

\section{Property of the two-terminal VSC-HVDC system}

After achieving the approximate symbolic expressions of the system poles, the impact of physical and control parameters can be evaluated in this section.

1) Trends of the real parts of the eigenvalues: From (25) and (19), it is known that if the roots are complex then the real part of the system eigenvalues are given as follows, where the approximation is based on the fact that the difference between terminal voltages are so small that they can be neglected compared with the other terms.

$$
\begin{aligned}
\lambda_{1}+\lambda_{2} & =2 * \operatorname{Re}\left\{\lambda_{1,2}\right\}=-0.5\left(a+d-e+2 R_{d c} / L_{d c}\right) \\
& =-\frac{1}{2}\left[\frac{C_{c}}{C_{t o t}}\left(a_{d}+a_{f}\right)-\frac{P_{20}}{C_{t o t}}\left(\frac{1}{v_{d c 10}^{2}}-\frac{1}{v_{d c 20}^{2}}\right)\right]-\frac{R_{d c}}{L_{d c}} \\
& \approx-\frac{C_{c}}{2 C_{t o t}}\left(a_{d}+a_{f}\right)-\frac{R_{d c}}{L_{d c}} \\
\lambda_{3}+\lambda_{4} & =2 * \operatorname{Re}\left\{\lambda_{3,4}\right\}=-0.5(a+d-e) \\
& =-\frac{1}{2}\left[\frac{C_{c}}{C_{t o t}}\left(a_{d}+a_{f}\right)-\frac{P_{20}}{C_{t o t}}\left(\frac{1}{v_{d c 10}^{2}}-\frac{1}{v_{d c 20}^{2}}\right)\right] \\
& \approx-\frac{C_{c}}{2 C_{t o t}}\left(a_{d}+a_{f}\right)
\end{aligned}
$$

It shows that:

i By increasing $a_{d}$ or $a_{f}$, all the system eigenvalues will move (to the left) away from the imaginary axis with the relevant rate of change $C_{c o n v} / C_{t o t} / 4$.

ii By increasing the cable length, the ratio of $C_{\text {conv }} / C_{\text {tot }}$ will decrease and thus the real part of all the system eigenvalues will decrease i.e. system eigenvalues move towards the imaginary axis. 
iii By increasing the ratio between cable resistance density and inductance density, the real part of the poorlydamped system eigenvalues will move (to the left) away from the imaginary axis. However, the real part of the well-damped system eigenvalues pair will not change dramatically.

iv Since the difference between terminal voltage are too small, the operating point of transmitted active power $P_{20}$ will not play an important role with respect to the real part of system eigenvalues.

2) Trends of the natural frequencies of the eigenvalues: From (25) and (19), it is known that if the roots are complex then the natural frequencies of the system eigenvalues are given as follows, where the approximation is based on the fact that the transmission losses $\left(R_{d c}\right)$ is much lower than the load $\left(v_{d c 20} / i_{d c 20}\right)$ :

$$
\begin{aligned}
\lambda_{1} \cdot \lambda_{2} & =\left|\lambda_{1,2}\right|^{2}=\omega_{n 1}^{2} \\
& =\frac{2}{L_{d c} C_{t o t}}\left(1-\frac{R_{d c} i_{d c 0}}{v_{d c 20}}\right) \approx \frac{2}{L_{d c} C_{t o t}} \\
\lambda_{3} \cdot \lambda_{4} & =\left|\lambda_{3,4}\right|^{2}=\omega_{n 2}^{2} \\
& =\frac{1}{2} \frac{C_{c}}{C_{t o t}} a_{d} a_{f}
\end{aligned}
$$

It shows that:

i The natural frequency of the poorly damped pole pair $\left(\omega_{n 1}\right)$ is dominated by the shunt capacitor and cable inductance. $\omega_{n 1}$ is decreased by increasing the total shunt capacitor $C_{t o t}$ or increasing the cable inductance.

ii For the well damped pole pair, if $C_{d c} \ll C_{c}$ and thus $C_{c} \approx C_{t o t}$, its natural frequency $\omega_{n 2}^{2} \approx a_{d} a_{f} / 2$.

3) Trends of the damping ratios of the eigenvalues: For a second order polynomial $\lambda^{2}+k_{1} \cdot \lambda+k_{2}=0$ with two complex roots, the characteristic frequency is $\omega_{0}=\sqrt{k_{2}-k_{1}^{2} / 4}$ and the damping ratio is $\zeta=k_{1} / 2 / \sqrt{k_{2}}$. It implies that by increasing $k_{1}$ and decreasing $k_{2}$, the system characteristic frequency decreases and the damping ratio increases and thus has better dynamic performance.

i For the poorly damped pole pair $\lambda_{1,2}$, we have:

$$
\begin{aligned}
k_{1} & \approx \frac{C_{c}}{2 C_{t o t}}\left(a_{d}+a_{f}\right)+\frac{R_{d c}}{L_{d c}} ; \quad k_{2} \approx \frac{2}{L_{d c} C_{t o t}} \\
\zeta_{1} & \approx\left[\frac{C_{c}}{2 C_{t o t}}\left(a_{d}+a_{f}\right)+\frac{R_{d c}}{L_{d c}}\right] \sqrt{\frac{L_{d c} C_{t o t}}{8}}
\end{aligned}
$$

We can increase the poorly damped system damping ratio by increasing the VSC shunt capacitor $C_{c}$ or the system bandwidths $a_{d}, a_{f}$. For fixed cable resistance $R_{d c}, \zeta_{1}$ decreases when $L_{d c}$ increases.

ii For the well damped pole pair $\lambda_{3,4}$, we have (assuming $\left.C_{d c} \ll C_{c}\right)$ :

$$
\begin{aligned}
& k_{1} \approx \frac{1}{2}\left(a_{d}+a_{f}\right) \quad k_{2} \approx \frac{1}{2} a_{d} a_{f} \\
& \zeta_{2} \approx \frac{a_{d}+a_{f}}{\sqrt{8 a_{d} a_{f}}}=\frac{1}{2} \sqrt{1+\frac{1}{2}\left(\frac{a_{d}}{a_{f}}+\frac{a_{f}}{a_{d}}\right)}
\end{aligned}
$$

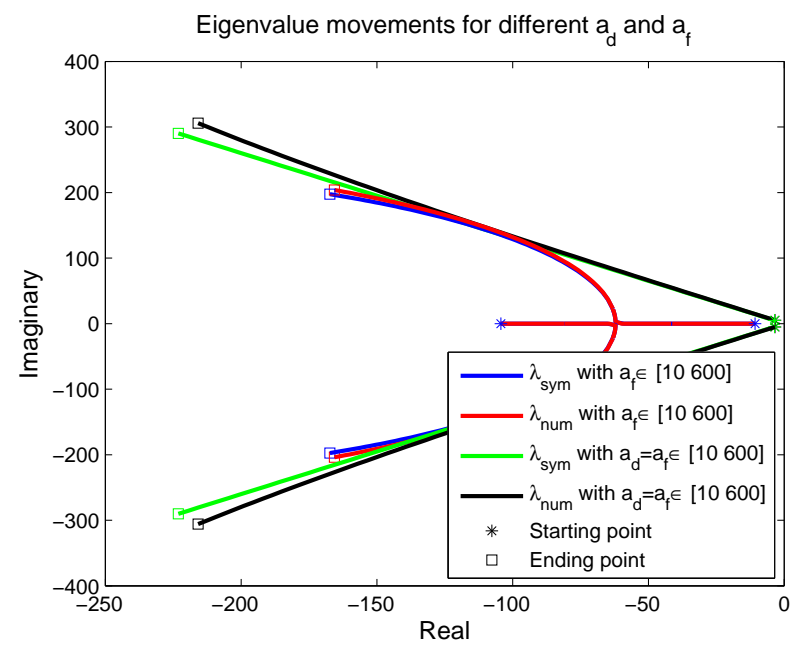

Fig. 2: Well damped eigenvalue movements for different $a_{d}$ and $a_{f}$

It is known that $a_{d} / a_{f}+a_{f} / a_{d} \geq 2$ where the equality holds when $a_{d}=a_{f}$. Therefore, $\zeta_{2} \geq 1 / \sqrt{2}$, and with larger difference between $a_{d}$ and $a_{f}$, the damping ratio of the well damped pole pair increases, which is illustrated in Fig.2,

\section{MULTI-TERMINAL VSC-HVDC SYSTEM}

In this section, the proposed method will be applied to an MTDC system. Assuming that, within an MTDC system, there is only one converter controlling the DC-voltage and the other converters control their transmitted active power between the AC- and DC-grid nodes.

The MTDC system can be described as: one DC-voltage controlled VSC with its shunt capacitor (2nd order system $G_{v s c}(s)$ ); one $R L C R_{0}$ DC-grid $\left(G_{d c}(s)\right)$, where $R_{0}$ describes the active power controlled VSC and, at converters steady states, $R_{k 0}=v_{d c k 0}^{2} / P_{k 0}$ and $k$ implies the $k$ th VSC. The block diagram of an autonomous MTDC system is depicted in Fig.3.

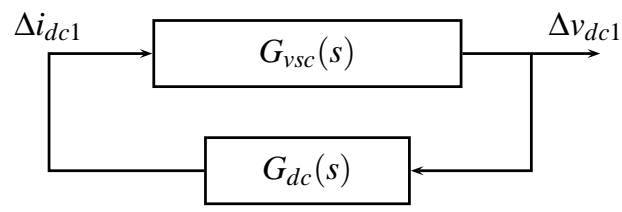

Fig. 3: Block diagram of the MTDC system with zero input signals

In addition, the parameters of the DC-grid are assumed to be fully known and the parameters at the DC-controlled VSC have free values. The purpose is then to understand the influence of the design bandwidths $\left(a_{d}, a_{f}\right)$, and the chosen shunt capacitor $\left(C_{c 1}\right)$ on the dynamics of $\Delta v_{d c 1}$ within an MTDC system.

\section{A. Symbolic-Isolation method}

If only the DC-voltage controlled VSC contains symbolic design parameters, we can treat it as a port and reduce the 
DC-grid by a standard model-reduction approach such as the Arnoldi Algorithm [17]. Since this model order reduction algorithm in the state space do not change the input/output port structure and only one port is needed, the isolated symbolic method [10] is simple and straight forward to apply here.

For the DC-grid dynamic system, the input signal is the voltage of the DC-voltage controlled VSC $\left(\Delta v_{d c 1}\right)$ and the output is the DC-current flow into the DC-grid $\left(\Delta i_{d c 1}\right)$. In order to apply the symbolic solution, obtained in the Sec.III, the DC-grid system order is reduced to the second order:

$$
\begin{aligned}
{\left[\begin{array}{l}
\dot{z}_{1} \\
\dot{z}_{2}
\end{array}\right] } & =\left[\begin{array}{ll}
a_{z 11} & a_{z 12} \\
a_{z 21} & a_{z 22}
\end{array}\right]\left[\begin{array}{l}
z_{1} \\
z_{2}
\end{array}\right]+\left[\begin{array}{l}
b_{z 1} \\
b_{z 2}
\end{array}\right] \Delta v_{d c 1} \\
\Delta i_{d c 1} & =\left[\begin{array}{ll}
c_{z 1} & c_{z 2}
\end{array}\right]\left[\begin{array}{l}
z_{1} \\
z_{2}
\end{array}\right]
\end{aligned}
$$

In order to make the 4th order state matrix of the reduced order MTDC system to have the same structure as $A_{s}$, a similarity transformation is required to the above 2 nd order system. The similarity matrix is $\mathrm{T}$, where $\tilde{z}=T z$ :

$$
\begin{aligned}
T & =\left[\begin{array}{cc}
c_{z 1} & c_{z 2} \\
k \cdot b_{z 2} & -k \cdot b_{z 1}
\end{array}\right] \\
k & =-\frac{a_{z 12} c_{z 1}^{2}-a_{21} c_{z 2}^{2}+c_{z 1} c_{z 2}\left(a_{z 22}-a_{z 11}\right)}{\left(b_{z 1} c_{z 1}+b_{z 2} c_{z 2}\right)^{2}}
\end{aligned}
$$

\section{B. Study case: three-terminal VSC-HVDC system}

The simulated system is presented in Fig. 4, where station 1 controls the DC-voltage and the remaining converters control the transmitted active power. Each of the DC-lines is modeled as a single $\Pi$-section with parameters given in Tab. I.

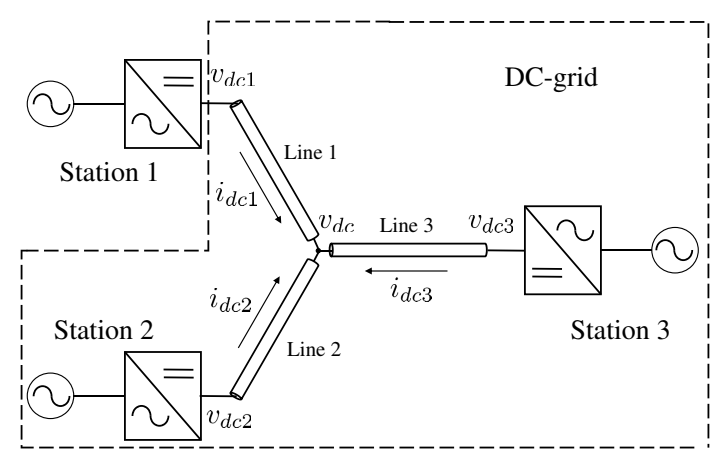

Fig. 4: Study case 1: 'Y'-shape three-terminal VSC-HVDC system

For fixed set points of $v_{d c 10}, P_{k 0}, k=2,3$ and cable length (which are shown in Tab. III), the equivalent resistances $R_{k 0}$ are countable, i.e. $R_{20}=-667[\Omega]$ and $R_{30}=-1003[\Omega]$

The linear circuit model can always be described by the following equations:

$$
\begin{aligned}
C_{g} \frac{\mathrm{d} x_{g}}{\mathrm{~d} t}+G_{g} x_{g} & =B_{g} u_{g} \\
y_{g} & =F_{g} x_{g}
\end{aligned}
$$

In our study case, the state space model matrices are given below, where $L_{d c k}, R_{d c k}, C_{d c k}$ are the inductance, resistance,
TABLE III: DC-grids dynamic parameters

\begin{tabular}{|c|c|}
\hline Property & Value \\
\hline Rated DC-voltage of station 1,2,3 & $640[\mathrm{kV}]$ \\
\hline Operating point of active power of station 2 & $-600[\mathrm{MW}]$ \\
\hline Operating point of active power of station 3 & $-400[\mathrm{MW}]$ \\
\hline Shunt capacitor of station 2,3 & $20[\mu \mathrm{F}]$ \\
\hline Distance of Line 1,2 & $100[\mathrm{~km}]$ \\
\hline Distance of Line 3 & $200[\mathrm{~km}]$ \\
\hline
\end{tabular}

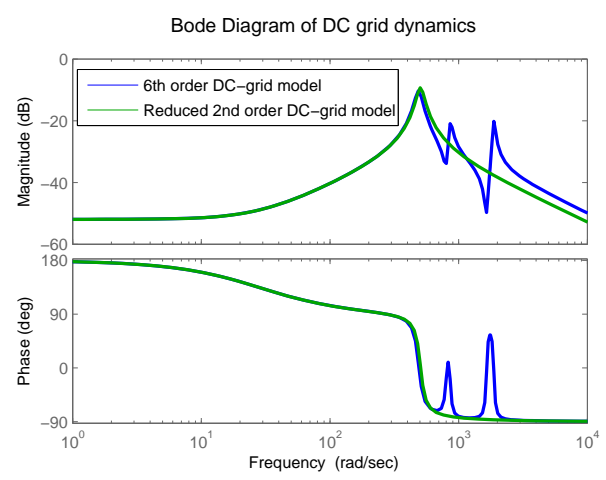

Fig. 5: Study case 1: Bode plot comparison

capacitance (one in each side of the cable) of the $k$-th line and $C_{c k}$ is the shunt capacitor of the $k$-th VSC.

$$
\begin{aligned}
& x_{g}^{T}=\left[\begin{array}{llllll}
i_{d c 1} & v_{d c} & i_{d c 2} & v_{d c 2} & i_{d c 3} & v_{d c 3}
\end{array}\right] ; \\
& u_{g}=v_{d c 1} ; \quad y_{g}=i_{d c 1} \text {; } \\
& C_{g}=\operatorname{diag}\left\{L_{d c 1}, C_{d c 1}+C_{d c 2}+C_{d c 3}, L_{d c 2}, C_{d c 2}+C_{c 2}\right. \text {, } \\
& \left.L_{d c 3}, C_{d c 3}+C_{c 3}\right\} \\
& G_{g}=\left[\begin{array}{cccccc}
R_{d c 1} & 1 & 0 & 0 & 0 & 0 \\
-1 & 0 & -1 & 0 & -1 & 0 \\
0 & 1 & R_{d c 2} & -1 & 0 & 0 \\
0 & 0 & 1 & \frac{1}{R_{20}} & 0 & 0 \\
0 & 1 & 0 & 0 & R_{d c 3} & -1 \\
0 & 0 & 0 & 0 & 1 & \frac{1}{R_{30}}
\end{array}\right] \quad B_{g}=\left[\begin{array}{l}
1 \\
0 \\
0 \\
0 \\
0 \\
0
\end{array}\right] \\
& F_{g}=\left[\begin{array}{llllll}
1 & 0 & 0 & 0 & 0 & 0
\end{array}\right]
\end{aligned}
$$

The poles of the above DC-grid system are located strictly in the left half plane. Therefore, the system in this study case is stable but not passive. (Note that the DC-grid involves active elements in contrast to the transmission line only.)

The Arnoldi Algorithm [17] is applied here to reduce the DC-grid dynamic system order into a 2nd order system. After similarity transformation with matrix $T$ (35), the numerical second order state space model is:

$$
\begin{aligned}
{\left[\begin{array}{l}
\dot{\tilde{z}}_{1} \\
\tilde{z}_{2}
\end{array}\right] } & =\left[\begin{array}{cc}
-94.8 & -22.8 \\
11315 & 28.3
\end{array}\right]\left[\begin{array}{l}
\tilde{z}_{1} \\
\tilde{z}_{2}
\end{array}\right]+\left[\begin{array}{c}
22.8 \\
0
\end{array}\right] \Delta v_{d c 1} \\
\Delta i_{d c 1} & =\left[\begin{array}{ll}
1 & 0
\end{array}\right]\left[\begin{array}{l}
\tilde{z}_{1} \\
\tilde{z}_{2}
\end{array}\right]
\end{aligned}
$$

The bode plot comparison is given in Fig. 5. It shows that the reduced 2 nd order model characterizes the original system well for $\omega<800[\mathrm{rad} / \mathrm{s}]$. 


\section{Extended method for multi-terminal VSC-HVDC system}

After the model order reduction of DC-grid dynamics, the state space model of the MTDC system is:

$$
\begin{aligned}
\frac{\mathrm{d}}{\mathrm{d} t} \hat{x} & =\hat{A}_{s} \cdot \hat{x}+\hat{B}_{s} \cdot \Delta v_{d c 1}^{r e f} \\
\hat{y} & =\hat{C}_{s} \cdot \hat{x}=\left[\begin{array}{llll}
0 & 1 & 0 & 0
\end{array}\right] \cdot \hat{x}=\Delta v_{d c 1} \\
\hat{x}^{T} & =\left[\begin{array}{llll}
\Delta i_{l o a d}^{f} & \Delta v_{d c 1} & \Delta \hat{i}_{\text {line }} & \Delta \hat{v}_{d c}
\end{array}\right] \\
\hat{A}_{s}= & {\left[\begin{array}{cccc}
-a_{f} \frac{C_{c}}{C_{t o t}} & a_{f} \frac{C_{c} i_{d c 10}-a_{d} C_{d c} C_{c} v_{d c 10}}{C_{t o t} v_{d c 10}} & a_{f} \frac{C_{c}}{C_{t o t}} & 0 \\
\frac{1}{C_{t o t}} & -\frac{a_{d} C_{c} v_{d c 10}+i_{d c 10}}{C_{t o t} v_{d c 10}} & -\frac{1}{C_{t o t}} & 0 \\
0 & 22.8 & -94.8 & -22.8 \\
0 & 0 & 11315 & 28.32
\end{array}\right] } \\
\hat{B}_{s}^{T} & =\left[\begin{array}{llll}
a_{f} a_{d} \frac{C_{d c} C_{c}}{C_{t o t}} & a_{d} \frac{C_{c}}{C_{t o t}} & 0 & 0
\end{array}\right]
\end{aligned}
$$

With the proposed method, the characteristic polynomial of $\hat{A}_{s}$ can be approximated as follows, where $a, b, d, e$ are defined in (17), $c_{1}$ is the shut capacitor at station 1 i.e. $c_{1}=C_{t o t}$ and in this study case, $c_{2}=88.4 \cdot 10^{-6}, R / L=94.8$, $e=28.32$ :

$$
\begin{aligned}
& \operatorname{det}\left(\lambda I-\hat{A}_{s}\right)=\hat{p}_{1}(\lambda) \hat{p}_{2}(\lambda) \\
& \hat{p}_{1}(\lambda)=\lambda^{2}+\left(\frac{c_{2}(a+d)-c_{1} e}{c_{1}+c_{2}}+\frac{R}{L}\right) \lambda+\frac{c_{1}+c_{2}}{c_{1}}\left(\frac{1}{L c_{2}}-e \frac{R}{L}\right) \\
& \hat{p}_{2}(\lambda)=\lambda^{2}+\frac{c_{1}(a+d)-c_{2} e}{c_{1}+c_{2}} \lambda+\frac{\left(a d c_{1}-b\right)}{c_{1}+c_{2}}
\end{aligned}
$$

Compared with the two-terminal VSC-HVDC system with parameter given in Tab. I, both the shunt capacitor at station 2 and the ratio of $L_{d c} / R_{d c}$ increase, implying that the damping ratio of the poorly damped pole pair will increase. In addition, the poorly damped poles move further away from the imaginary axis and the well damped poles move towards the imaginary axis. The numerical result for the system poles is given in Tab. IV, which as well supports our conclusion. Consequently, the MTDC has better damping performance compared with the the two terminal VSC-HVDC system for the poorly damped pole pair but worse damping performance for the well damped pole pair.

TABLE IV: DC-grids dynamic parameters

\begin{tabular}{|c|c|c|c|c|}
\hline Study case & \multicolumn{4}{|c|}{ Eigenvalues } \\
\hline Two-terminal & $-158+1511 \mathrm{i}$ & $-158-1511 \mathrm{i}$ & $-110+147 \mathrm{i}$ & $-110-147 \mathrm{i}$ \\
\hline \multirow{2}{*}{ MTDC } & $-66+781 \mathrm{i}$ & $-66-781 \mathrm{i}$ & $-77-1972 \mathrm{i}$ & $-77+1972 \mathrm{i}$ \\
& $-178+1061 \mathrm{i}$ & $-178-1061 \mathrm{i}$ & $-48+112 \mathrm{i}$ & $-48-112 \mathrm{i}$ \\
\hline 4th order MTDC & $-254+1037 \mathrm{i}$ & $-254-1037 \mathrm{i}$ & $-48+112 \mathrm{i}$ & $-48-112 \mathrm{i}$ \\
\hline
\end{tabular}

\section{CONCLUSIONS}

This paper provides an analytical method to describe a simplified two-terminal VSC-HVDC system in terms of purely symbolic eigenvalue expressions. For a wide variation of the physical and control parameters of the investigated VSC-HVDC system, the method achieves to provide compact expressions that fit the numerically calculated eigenvalues of the system, with relatively small errors. Accordingly, it has been possible to provide pole movements with the impact of different physical and control parameters.

In addition, by applying the symbolic-isolation method, the order of a multi-terminal VSC-HVDC system can be reduced to an equivalent two-terminal VSC-HVDC system, which enables the proposed method to provide symbolic pole expressions. It shows that within some MTDC systems, the damping performance of the poorly damped pole pair improves.

\section{REFERENCES}

[1] N. Flourentzou, V. Agelidis, and G. Demetriades, "VSC-based HVDC power transmission systems: An overview," IEEE Trans. Power Electronics, vol. 24, no. 3, pp. 592 - 602, March 2009.

[2] M. Bahrman, E.V. Larsen, R.J. Piwko, and H.S. Patel, "Experience with HVDC - turbine-generator torsional interaction at square butte," IEEE Trans. Power App. Syst., vol. 99, no. 3, pp. 966 - 975, May 1980.

[3] N. Prabhu, and K. R. Padiyar, "Investigation of subsynchronous resonance with VSC-Based HVDC transmission systems," IEEE Trans. Power Delivery, vol. 24, no. 1, pp. 433 - 440, Jan. 2009.

[4] S.D. Sudhoff, S.F. Glover, P.T. Lamm, D.H. Schmucker, and D.E. Delisle, "Admittance space stability analysis of power electronic systems," IEEE Trans. Aerosp. Electron. Syst., vol. 36, no. 3, pp. 965 - 973, July 2000.

[5] C. Wan, M. Huang, C.K. Tse, and X. Ruan, "Stability of interacting grid-connected power converters," Journal of Modern Power Systems and Clean Energy, Springer, vol.1, no.3, pp. 249 - 257, Dec. 2013.

[6] G.O. Kalcon, G.P. Adam, O. Anaya-Lara, S. Lo and K. Uhlen, "Smallsignal stability analysis of multi-terminal VSC-based DC transmission systems," IEEE Trans. Power Syst., vol. 27, no. 4, pp. 1818 - 1830, Nov. 2012.

[7] T. Tanaka, S. Fujikawa and S. Funabiki, "A new method of damping harmonic resonance at the DC link in large-capacity rectifier-inverter systems using a novel regenerating scheme," IEEE Trans. Ind. Appl., vol. 38, no. 4, pp. 1131 - 1138, July-August 2002.

[8] L. Zhang, L. Harnefors and H. P. Nee, "Interconnection of two very weak AC systems by VSC-HVDC links using power-synchronization control," IEEE Trans. Power Syst., vol. 26, no. 1, pp. 344 - 355, Feb. 2011.

[9] L. Harnefors, N. Johansson, L. Zhang and B. Berggren, "Interarea oscillation damping using active-power modulation of multiterminal HVDC transmissions," IEEE Trans. Power Syst., vol. 29, no. 5, pp. 2529 - 2538, Sept. 2014.

[10] G. Shi, B. Hu, and C. J. Richard Shi, "On symbolic model order reduction," IEEE Trans. computer-aided design of intergrated circuits and systems, vol. 25, no. 7, pp. 1257 - 1272, Jul. 2006.

[11] Y. Song and C. Breitholtz, "Nyquist Stability Analysis of an AC-grid Connected VSC-HVDC System Using a Distributed Parameter DCcable Model," to appear in IEEE Trans. Power Delivery.

[12] B. T. Ooi and X. Wang, "Voltage angle lock loop control of the boost type PWM converter for HVDC application," IEEE Trans. Power Electronics, vol. 5, no. 2, pp. 229 - 235, April 1990.

[13] L. Harnefors, M. Bongiorno, and S. Lundberg, "Input-Admittance calculation and shaping for controlled voltage-source converters," IEEE transactions on industrial electronics, vol. 54, no. 6, pp. 3323 - 3334, 2007.

[14] N. Mohan, Advanced electric drives: analysis, control and modeling using Simulink, MNPERE, 2001.

[15] B. K. Bose, Power electronics and variable frequency drives, New York: IEEE Press, 1997.

[16] Y. Song and C. Breitholtz, "Nyquist stability analysis of a VSC-HVDC system using a distributed parameter DC-cable model," in 19th World Congress of the International Federation of Automatic Control, Cape town, South Africa, August 2014.

[17] I. M. Jaimoukha, "A general minimal residual Krylov subspace method for large-scale model reduction," in IEEE Transactions on Automatic Control, vol. 42, no. 10, pp. 1422 - 1427, Oct 1997. 